\title{
¿Cómo practican los psicólogos la psicoterapia? Estudio fenomenológico de profesionales venezolanos
}

\author{
María Campo-Redondo리 , Evelyn M. García ${ }^{2}$ y Laura Gamboa ${ }^{3}$ \\ Universidad de los Emiratos Árabes Unidos/Universidad del Zulia/Universidad Rafael Urdaneta. \\ Emiratos Árabes Unidos/Venezuela
}

\begin{abstract}
La presente investigación tuvo como objetivo caracterizar el concepto de psicoterapia en psicólogos venezolanos, partiendo de la entrevista que se hiciera a 7 psicólogos clínicos, con distintos enfoques teóricos, y con una experiencia en dicha práctica entre 3 a 37 años. Se empleó la Teoría Fundamentada para organizar los datos. Luego de realizar el análisis, los resultados arrojaron que los entrevistados conciben a la psicoterapia como un proceso basado en una relación que conduce a un crecimiento personal, tanto del paciente como del terapeuta; así mismo, se determinó que el éxito del proceso psicoterapéutico depende del establecimiento de una buena alianza terapéutica porque favorece el surgimiento de aspectos íntimos y subjetivos del paciente. Como coordenada central, se obtuvo que la psicoterapia basada en la relación favorece al paciente y al proceso terapéutico, y está omnipresente en las prácticas de los profesionales entrevistados, ya que los venezolanos son vinculares por razones culturales. Como conclusión, se destaca la importancia de incorporar en el proceso de formación de psicoterapeutas, características antropológicas encontradas por autores en Venezuela sobre el modo de vida relacional del venezolano, y esta relacionalidad no escapa a los psicólogos que practican psicoterapia.
\end{abstract}

Palabras clave: Concepto de psicoterapia, psicólogos clínicos, Teoría Fundamentada

This research was aimed to characterize the concept of psychotherapy in Venezuelan clinical psychologists. We interviewed 7 clinical psychologists with different theoretical approaches and experience in psychotherapy between 3 and 37 years. The analysis was performed under the method of Grounded Theory. After performing the analysis, the results showed that psychotherapy is a process based on the relationship that leads to a personal growth and psychotherapy depends on establishing a good therapeutic alliance that favors the emergence of intimate and subjective aspects of the patient, also generating personal growth in the therapist. Finally, the psychotherapy based on the relationship helps the patient and the therapeutic progress, because Venezuelans are "relational" by cultural reasons. As conclusion, it is highlighted the importance of inclusion of the anthropological characteristics that Venezuelan authors have pointed out in the relational way of life of Venezuelan people, and this includes also to those psychologists who practice psychotherapy

Key Words: concept of psychotherapy, clinical psychologists, Grounded Theory.

English Title: How do psychologists practice psychotherapy?. Phenomenological study of Venezuelan professionals

\section{Cita bibliográfica / Reference citation:}

Campo Redondo, M., García, E.M. y Gamboa, L. (2019). ¿Cómo practican los psicólogos la psicoterapia?. Estudio fenomenológico de profesionales venezolanos. Clínica e Investigación Relacional, 13 (2): 455-476. [ISSN 1988-2939] [Recuperado de www.ceir.info ] DOI: $10.21110 / 19882939.2019 .130209$

\footnotetext{
${ }^{1}$ Psicóloga. Doctora en Ciencias Humanas. Profesora de la Universidad de los Emiratos Árabes Unidos y Emérita de la Universidad de Zulia. mcampo@uaeu.ac.ae

${ }^{2}$ Psicóloga. Magister en Psicología Clínica. Ministerio del Poder Popular para la Educación. Cabimas, Venezuela. evegarcia2703@gmail.com

3 Psicóloga. Magister en Psicología Clínica. Universidad Rafael Urdaneta y Universidad Rafael María Baralt. Maracaibo/Cabimas, Venezuela.gamboarivas@hotmail.com, lauraiseley@gmail.com.
} 


\section{Introducción}

Hoy en día se considera la psicoterapia como una alternativa válida de tratamiento de los problemas psíquicos o psiquiátricos, tan importante como los psicofármacos, y se ha convertido en una práctica socialmente aceptada y necesaria. Se pudiera decir que en algunos países es un objeto de consumo masivo, sin embargo, su lugar dentro de la ciencia no ha estado exenta de cuestionamientos.

En este trabajo, se entrelazan dos conceptos, psicología clínica y psicoterapia, que, aunque relacionados entre sí, tienen epigénesis distintas. Para estudiar estas dos vertientes, se ubicó un espacio concreto de ejercicio: La práctica de la psicoterapia definida por psicólogos clínicos en Venezuela. ${ }^{1}$

Con el fin de responder a la pregunta central, a saber, cómo conciben y practican la psicoterapia los profesionales en Venezuela, se entrevistó a 7 psicólogos clínicos sobre la conceptualización que ellos han hecho de esta intervención terapéutica en sus lugares de trabajo. Interesó en particular, abordar sus experiencias al practicar psicoterapia como medio de vida, y registrar sus palabras textuales; de ahí que se escogiera un enfoque cualitativo y fenomenológico, para que, inductivamente, es decir, desde los datos concretos y textuales, se pudiese pasar a una elaboración de las coordenadas teóricas que definen a la psicoterapia, realizada por los propios practicantes en Venezuela.

Varios autores (por ejemplo, Lilliengren y Werbart, 2010) han argumentado que los terapeutas a lo largo de su experiencia practicando psicoterapia, desarrollan modelos internos (y privados) a la hora de abordar su práctica. A estos modelos también se les ha descrito como "teorías implícitas del terapeuta", y estas "teorías" se entremezclan con la orientación teórica explícita sobre cómo llevar a cabo las intervenciones clínicas. Las teorías implícitas son una mezcla de experiencias personales, entrenamiento formal y reflexión profesional y pueden incluir las estrategias personales del terapeuta de lo que se debe hacer, así como aquello que NO debe hacerse en psicoterapia. Estas estrategias personales, se han denominado "teorías de acción terapéutica", y podrían resumirse como aquellas ideas que el terapeuta ha elaborado sobre lo que él cree que funciona (o no) en psicoterapia. Los autores antes mencionados, han afirmado que las teorías implícitas de la acción terapéutica pueden ser superiores a las teorías explicitas, en cuanto a definir las acciones llevadas a cabo por los practicantes de la psicoterapia.

En tal sentido, estudiar la teorización implícita de los terapeutas puede contribuir a la comprensión de lo que es útil y lo que impide el tratamiento con poblaciones de pacientes particulares. Esta es en concreto, la pregunta general formulada en esta investigación, a saber, cuáles son las "teorías implícitas de la acción terapéutica" y cómo han influido estas 
teorías en la conceptualización que hacen de la psicoterapia los psicólogos clínicos venezolanos.

El presente artículo presenta una contextualización del fenómeno estudiado, se describen los procedimientos metodológicos, se analizan los resultados y se presentan algunas ideas concluyentes, dejando abierto el camino para futuras investigaciones de corte más estadístico y generalizable.

\section{Psicología Clínica y psicoterapia}

La psicología clínica se puede delimitar como la rama de la ciencia psicológica que aborda problemas relativos al área de la salud mental, donde las acciones de evaluación, diagnóstico y tratamiento (o intervención) son el norte. La palabra "clínica" (klinós) significa cama; el clínico es aquel quien se inclina sobre el que yace en la cama, (quien padece), y la orientación clínica en psicología se interesa por quien tiene problemas mentales y conductuales. De este modo, la psicología clínica estudia al individuo con trastornos cognitivos, emocionales y/o conductuales, que tengan o no una base biológica (Amaro, Guasch y Sarlé 2007).

Por otro lado, la palabra terapia o terapéutica viene del griego "terapeytikee" y del verbo "terapeyo", y se traduce en dos acciones: servir y cuidar. El termino psicoterapia, está íntimamente relacionado con el de intervención en psicología clínica. La práctica de la psicoterapia incluye el tratamiento de los trastornos psíquicos, por medios psicológicos, con el objetivo de generar un cambio favorable en quien se denomina paciente, y que trasciende los límites del conocimiento científico. La palabra intervención, propia de la psicología clínica, significa mediación, ponerse en medio, inter=entre, venir=situarse, actuar entre las personas, y esta dimensión de intervención se asocia fundamentalmente con la psicoterapia (Amaro et al, 2007).

Resulta interesante remarcar que la definición de psicoterapia, lleva implícita un encuentro entre dos sujetos, que, aunque con roles distintos y asimétricos, ambos participantes están implicados en el acto. Se puede decir entonces que el suceder terapéutico es un acontecimiento intersubjetivo, y por tal motivo, tan necesario es estudiar al paciente, como las variables y "voces" del terapeuta. De esta manera, las emociones, pensamientos, y comportamientos emergentes en el terapeuta se pueden utilizar como un elemento de análisis y de intervención.

\section{Psicoterapia en Venezuela}

En Venezuela, la psicoterapia como actividad profesional es relativamente joven, aunque los profesionales que suelen ejercer esta práctica, a saber, principalmente psicólogos, la vienen realizando desde la década del 60 del siglo pasado (Rodríguez y Sánchez, 1996); sin 
embargo, no fue sino hasta finales de los 9o, cuando los psicoterapeutas de Venezuela se organizaron bajo la Asociación Venezolana de Psicoterapia (AVEPSI), para darle visibilidad y orden ético a esta práctica. Así, AVEPSI nació en el año 1999, ante la necesidad de crear una asociación nacional, no existente hasta el momento en el país, para agrupar al mayor número de psicoterapeutas, y contribuir a la definición de condiciones claras para el ejercicio de la psicoterapia como profesión. AVEPSI surgió apoyándose en la Declaración de Estrasburgo en Materia de Psicoterapia (recuperado de AVEPSI http://avepsi.org.ve/site/laasociacion/historia-de-avepsi). ${ }^{2}$

A pesar de los esfuerzos titánicos de AVEPSI para darle visibilidad a la psicoterapia, las investigaciones sobre esta práctica son casi inexistentes en Venezuela, en especial aquellas relacionadas con el psicoterapeuta como parte integrante fundamental del proceso.

En Venezuela no existe una línea de investigación relacionada con la conceptualización de la práctica psicoterapéutica realizada desde los propios practicantes, y mucho menos sobre las teorías implícitas de la acción terapéutica asociadas a esta práctica.

Relacionado con las ideas del párrafo anterior, es importante destacar que otros países de la región, como Colombia, por ejemplo, han emprendido una exploración del tema que compete a este estudio. En este sentido, Gómez (2010) realizó una investigación de tipo documental, para precisar los antecedentes históricos que dieron origen al concepto de psicoterapia, así como delimitar el concepto en psicología clínica. Sin embargo, la investigación la realiza desde una óptica documental y bibliográfica, no considerando las voces de los propios practicantes. Aun así, su propuesta da una idea de la importancia del tópico.

Siguiendo este orden de ideas, López (2010) remarca que, en condición de practicante, el terapeuta debe estar atento a cómo construye su estilo particular de proceder. En tal sentido, es importante que preste atención a 3 dominios fundamentales: a las emociones generadas por los diversos pacientes y procesos, a las frustraciones a las que se puede enfrentar, y a las enseñanzas de los diversos maestros enterados de su actuar. Todo esto con el fin de generar constantemente un autoconocimiento verdadero, para reconocer errores, debilidades, fortalezas y aciertos, y, sobre todo, para crecer como sujeto y psicoterapeuta.

La escasez de producción científica en torno al tema de investigación en el proceso de conceptualización terapéutico en Venezuela y la responsabilidad que el trabajo de intervención clínica en salud mental supone impulsan la indagación del tema que emprende esta investigación. 


\section{Objetivos de la investigación}

Se considera pertinente dar un primer paso y plantear, como objetivo general, explorar cómo los psicólogos clínicos de diferentes orientaciones representativas del trabajo psicoterapéutico conceptualizan, en sus propias palabras, criterios para definir las coordenadas de los tratamientos que imparten. Así mismo, se busca conocer el modo en que dichos criterios se encuentran organizados, así como el impacto que esta práctica tiene en sus practicantes. En consecuencia, se rastrean las coordenadas que orientan las construcciones que hace el psicólogo clínico venezolano sobre la psicoterapia; se explora qué tan efectiva es para estos practicantes esta práctica; además, se indaga qué determina el éxito de la psicoterapia, según sus propias experiencias ejerciendo esta actividad, qué papel tiene la personalidad del terapeuta, y qué pudiera ser iatrogénico en esta acción profesional.

El afán de explorar, junto con la ausencia de referentes sobre el tema en Venezuela, conduce a situar el interés en la información que los mismos profesionales pueden proporcionar sobre su labor clínica. Este primer paso puede significar un conocimiento valioso para el área de investigación en psicoterapia. A partir de él, se puede reflexionar y plantear la tarea conjunta de impulsar esta línea de investigación en el País. Así mismo, a partir de los resultados preliminares de esta investigación, se podrá implementar la conceptualización de la psicoterapia en los planes de estudio de pre y postgrado de psicología, tomando en consideración las características propias de la "venezolanidad" en la práctica de la psicoterapia.

\section{ASPECTOS METODOLÓGICOS}

Para llevar a cabo este estudio, se escogió la modalidad del enfoque cualitativo, puesto que la temática a investigar es indagatoria (más que correlacional o comprobatoria), buscando una comprensión holística y naturalista, centrada en la perspectiva interpretativa del significado que le otorgan los informantes a la pregunta central.

El análisis de los datos se realizó tomando en consideración los lineamientos de la Teoría Fundamentada (Strauss y Corbin, 2002), debido a que genera información compleja e inductiva de procesos psicológicos y subjetivos, como emociones y pensamientos, que son únicos y personales, y a los que no se accede de forma adecuada mediante métodos cuantitativos. Este abordaje es coherente con el estado del arte en la investigación sobre la psicoterapia en el psicólogo clínico venezolano, en atención a la escasa bibliografía sobre el tema y la necesidad de dar una base a futuras investigaciones. 


\section{Elección de Informantes Claves}

En la elaboración de este trabajo, se siguió el precepto de elegir a los informantes clave, escogidos de modo intencional, partiendo del interés de la investigación en consideración de un procedimiento general descrito por Strauss y Corbin, (2002) como muestreo teórico. Estos autores establecen que se busca descubrir significados a través de la interpelación profunda, en este caso a psicólogos clínicos y la conceptualización que ellos hacen de la psicoterapia. Se escogió a psicólogos clínicos, debido a que en Venezuela estos profesionales son los que usualmente emplean psicoterapia en sus modos de intervención (aunque como se mencionó anteriormente, los psicólogos no son los únicos profesionales que llevan a cabo estas labores). Estas personas aportaron una información inicial, que luego fue interrogada, mediante preguntas abiertas, pero previamente delineadas, en función de los objetivos de la investigación. Del análisis de los textos se derivaron categorías, subcategorías, y sus relaciones y se construyó la Teoría Fundamentada con los significados obtenidos de los mismos informantes. La Teoría Fundamentada exige que los informantes se seleccionen en la medida en que proporcionen mayor variabilidad, respecto al informante inicial.

En esta investigación se pudo encontrar la saturación mediante la recolección de datos en siete sujetos $(\mathrm{M}=7)$. Se escogieron psicólogos clínicos de variadas posturas y formaciones teóricas (empíricamente soportadas en la evidencia), con un rango de experiencia clínica de 3 a 37 años, todos con licencia para el ejercicio de la psicología en Venezuela, con denominación clínica, y practicantes de la psicoterapia. La tabla 1 describe la composición de los informantes clave y las características de las entrevistas realizadas.

Tabla 1 - Composición de los informantes clave y características de las entrevistas

\begin{tabular}{|l|l|l|l|l|l|l|l|l|}
\hline Nombre & Edad & Sexo & Profesión & Enfoque & $\begin{array}{l}\text { Años de } \\
\text { Experiencia }\end{array}$ & $\begin{array}{l}\text { Lugar de la } \\
\text { entrevista }\end{array}$ & $\begin{array}{l}\text { No de } \\
\text { Sesiones }\end{array}$ & $\begin{array}{l}\text { Duración } \\
\text { de } \\
\text { entrevista } \\
\text { la }\end{array}$ \\
\hline E1 & $\begin{array}{l}55 \\
\text { años }\end{array}$ & $\mathrm{F}$ & Psicóloga & $\begin{array}{l}\text { Psicoterapia } \\
\text { Relacional }\end{array}$ & 32 & Consultorio & 1 & $51: 46$ min \\
\hline E2 & $\begin{array}{l}42 \\
\text { años }\end{array}$ & $\mathrm{F}$ & Psicóloga & $\begin{array}{l}\text { Sistémico- } \\
\text { Interpersonal }\end{array}$ & 20 años & $\begin{array}{l}\text { Universidad } \\
\text { Rafael } \\
\text { Urdaneta } \\
\text { (URU) }\end{array}$ & 1 & $\begin{array}{l}1 \\
23 \text { min }\end{array}$ \\
\hline E3 & $\begin{array}{l}28 \\
\text { años }\end{array}$ & $\mathrm{F}$ & Psicóloga & $\begin{array}{l}\text { Psicología } \\
\text { Positiva }\end{array}$ & 7 años & $\begin{array}{l}\text { Universidad } \\
\text { Rafael } \\
\text { Urdaneta } \\
\text { (URU) }\end{array}$ & 1 & $48: 47$ min \\
\hline E4 & $\begin{array}{l}26 \\
\text { años }\end{array}$ & $\mathrm{F}$ & Psicóloga & Psicoanalítico & 3 años & $\begin{array}{l}\text { Universidad } \\
\text { Rafael }\end{array}$ & 1 & $46: 30 \mathrm{~min}$ \\
\hline
\end{tabular}




\begin{tabular}{|l|l|l|l|l|l|l|l|l|}
\hline & & & & & $\begin{array}{l}\text { Urdaneta } \\
\text { (URU) }\end{array}$ & & \\
\hline E5 & $\begin{array}{l}60 \\
\text { años }\end{array}$ & F & Psicóloga & $\begin{array}{l}\text { Psicoterapia } \\
\text { Relacional }\end{array}$ & 37 años & $\begin{array}{l}\text { Universidad } \\
\text { Rafael } \\
\text { Urdaneta } \\
\text { (URU) }\end{array}$ & 1 & $\begin{array}{l}1 \text { hora } \\
24 \mathrm{~min}\end{array}$ \\
\hline E6 & $\begin{array}{l}56 \\
\text { años }\end{array}$ & $\mathrm{F}$ & Psicóloga & Gestáltico & 33 años & $\begin{array}{l}\text { Universidad } \\
\text { Rafael } \\
\text { Urdaneta } \\
\text { (URU) }\end{array}$ & 2 & $\begin{array}{l}-1: 10 \text { hrs } \\
-30 \mathrm{~min}\end{array}$ \\
\hline E7 & $\begin{array}{l}50 \\
\text { años }\end{array}$ & $\mathrm{M}$ & Psicólogo & Constructivista & 27 años & $\begin{array}{l}\text { Universidad } \\
\text { Rafael } \\
\text { Urdaneta } \\
\text { (URU) }\end{array}$ & 1 & 48 min \\
\hline
\end{tabular}

Técnica de recolección de la información: La entrevista

Debido al interés de esta investigación, a saber, el concepto de psicoterapia en el psicólogo clínico venezolano, se eligió como técnica de recolección de datos a la entrevista con preguntas abiertas (pero con foco en el tema central de la investigación), en formato presencial ("cara a cara") e individual. Se tuvieron un total de 8 encuentros, con una duración total de aproximadamente 464 minutos de entrevista. En el anexo No 1 se presenta una muestra de las preguntas más repetidas.

Previo a cada entrevista, se contactó vía e-mail a cada uno de los informantes, y se les proporcionó un formulario explicativo (incluido los aspectos éticos, de confidencialidad y derecho a suspender la colaboración en cualquier momento del proceso investigativo), solicitando su participación y consentimiento.

\section{Técnica de registro de la información}

La información se registró usando la grabadora de un equipo móvil, se hicieron pruebas previas para asegurar el registro y se tomaron las previsiones para realizar la entrevista completa sin interrupciones; posteriormente se procedió a la detallada y minuciosa transcripción de las grabaciones, registrando interjecciones, pausa (mediante puntos suspensivos), y se anotaron gestos, expresiones emocionales y otras conductas de los informantes durante la entrevista.

\section{Método para la interpretación de la información}

Una vez transcrito el texto, los investigadores interrogaron lo más detalladamente posible cada una de las expresiones presentes y se compararon constantemente para dar profundidad al análisis. 
La creación de temas y subtemas se realizó de forma manual, en vez de mediante el uso de software de codificación. Esta decisión refleja el deseo de los investigadores en obtener una comprensión detallada de los datos y mostrar un respeto a los significados textuales de las verbalizaciones de los informantes.

\section{Codificación abierta de la información}

En este proceso se separó la información en ideas claras y completas que provinieron del mismo texto. Luego se nombraron las categorías representativas para su organización.

\section{Codificación axial de la información}

En esta codificación se reagruparon las categorías mediante sus relaciones, de manera que, si antes se separó cada una de ellas, ahora se vinculan de manera significante. Este paso conllevó a la elaboración de la teoría presente en los datos.

\section{Codificación selectiva}

La codificación selectiva identificó la categoría central y las subsidiarias se organizaron en torno a la principal. El trabajo de codificación se graficó y derivó en la obtención de las coordenadas de la teoría.

\section{Descripción del proceso de triangulación}

Una vez transcrito el texto, los investigadores interrogaron lo más detalladamente posible cada una de las expresiones, y se compararon constantemente para dar profundidad al análisis. Esto se realizó para generar mayor validez y encuentro con "la verdad" del texto analizado, y minimizar las preconcepciones teóricas y conjeturas personales de los propios investigadores. Strauss y Corbin (2002), plantean explícitamente la necesidad de trabajar en equipos de investigación, de manera que se pueda discutir y abundar en preguntas enriquecedoras del análisis. Esto se alcanzó en esta investigación a través del proceso de triangulación, y tuvo como objeto disminuir el posible sesgo subjetivo de los investigadores y aumentar la validez de los resultados. En el presente estudio se sometió las transcripciones al análisis de expertos. Se realizó la lectura de cada entrevista, y los expertos hicieron un análisis y verificación de las posibles categorías resultantes. La tabla 2 describe los profesionales que participaron en la triangulación.

Tabla 2 - Descripción de los jueces-expertos que participaron en la triangulación

\begin{tabular}{|ll|l|}
\hline \multicolumn{2}{|c|}{ Profesión del experto } & Experticia \\
\hline 1. & Psicólogo & $\begin{array}{l}\text { Especialista en Sexología, Docente Universitario de la Universidad Rafael } \\
\text { Urdaneta }\end{array}$ \\
\hline 2. & $\begin{array}{l}\text { Psicóloga, } \\
\text { Sexóloga }\end{array}$ & Formación teórica en Psicoanálisis Lacaniano \\
\hline
\end{tabular}




\begin{tabular}{|ll|l|}
\hline 3. & Psicólogo & Formación en Sexualidad \\
\hline 4. & Psicóloga & Licenciada en Comunicación Social \\
\hline 5. & Psicóloga Clínica & Docente Universitaria de la Universidad Rafael Urdaneta \\
\hline
\end{tabular}

\section{Análisis de contenido}

El análisis se inició con el microanálisis y las comparaciones sucesivas, estrictamente desde los datos o verbalizaciones aportados por los 7 informantes, hasta obtener la categoría central. Después, estas categorías fueron integradas en un gráfico que ejemplifica las relaciones entre ellas, así como los vínculos conceptuales que las conectan de manera indirecta (Figura 1).

\section{Análisis Descriptivo y categorización de la información}

En función a los datos obtenidos, se construyó la categoría central y las subcategorías que caracterizan el concepto de psicoterapia en los psicólogos clínicos venezolanos. La tabla 3 da cuenta de esta categorización.

\section{Tabla 3 - Categorías, definiciones y propiedades}

\begin{tabular}{|c|c|c|c|}
\hline Categorias & Nombre & Definición & Propiedades \\
\hline \multirow[t]{4}{*}{ Categoría Central } & \multirow{4}{*}{$\begin{array}{l}\text { La psicoterapia es un } \\
\text { proceso basado en un } \\
\text { tipo especial de } \\
\text { relación que conduce a } \\
\text { un crecimiento } \\
\text { personal }\end{array}$} & \multirow{4}{*}{$\begin{array}{l}\text { Es un proceso que se logra a } \\
\text { través del establecimiento de } \\
\text { una especial relación, que } \\
\text { permite acceder a la } \\
\text { subjetividad del paciente y } \\
\text { que genera un crecimiento } \\
\text { personal del propio } \\
\text { terapeuta. }\end{array}$} & $\begin{array}{l}\text { Es un proceso de acompañamiento, } \\
\text { para el crecimiento personal del } \\
\text { paciente. }\end{array}$ \\
\hline & & & $\begin{array}{l}\text { Depende del establecimiento de una } \\
\text { buena alianza terapéutica. }\end{array}$ \\
\hline & & & $\begin{array}{l}\text { Favorece el surgimiento de aspectos } \\
\text { íntimos y subjetivos del paciente }\end{array}$ \\
\hline & & & $\begin{array}{l}\text { Genera crecimiento personal en el } \\
\text { terapeuta }\end{array}$ \\
\hline \multirow[t]{4}{*}{ Sub- Categoría } & \multirow{4}{*}{$\begin{array}{l}\text { La psicoterapia } \\
\text { depende del } \\
\text { establecimiento de una } \\
\text { buena alianza } \\
\text { terapéutica. }\end{array}$} & \multirow{4}{*}{$\begin{array}{l}\text { Es una relación que está } \\
\text { basada en el establecimiento } \\
\text { de un vínculo y una alianza } \\
\text { que permite conocer al } \\
\text { paciente. }\end{array}$} & Genera una conexión profunda. \\
\hline & & & $\begin{array}{l}\text { Permite que el paciente se muestre } \\
\text { a profundidad. }\end{array}$ \\
\hline & & & Está basada en la empatía. \\
\hline & & & $\begin{array}{l}\text { Permite la transformación o cambio } \\
\text { en el paciente. }\end{array}$ \\
\hline \multirow[t]{4}{*}{ Sub- Categoría } & \multirow{4}{*}{$\begin{array}{l}\text { La psicoterapia } \\
\text { favorece el surgimiento } \\
\text { de aspectos íntimos y } \\
\text { subjetivos del paciente. }\end{array}$} & \multirow{4}{*}{$\begin{array}{l}\text { Es un espacio donde llega la } \\
\text { persona a consultar o buscar } \\
\text { ayuda a causa de problemas } \\
\text { o padecimientos que lo } \\
\text { conlleva al sufrimiento, y es } \\
\text { en ese proceso donde esta } \\
\text { paciente muestra su ser y a } \\
\text { su vez permite conocerse a sí } \\
\text { mismo y así poder construir } \\
\text { un cambio o transformación } \\
\text { en su vida. }\end{array}$} & Siente la necesidad de buscar ayuda. \\
\hline & & & $\begin{array}{l}\text { Permite al Terapeuta conocer las } \\
\text { vulnerabilidades del paciente. }\end{array}$ \\
\hline & & & El paciente hace introspección. \\
\hline & & & $\begin{array}{llr}\text { Es un proceso } & \text { que } & \text { genera } \\
\text { transformaciones } & \text { o } & \text { cambios } \\
\text { positivos. } & & \\
\end{array}$ \\
\hline
\end{tabular}




\begin{tabular}{|c|c|c|c|}
\hline \multirow[t]{5}{*}{ Sub- Categoría } & \multirow[t]{5}{*}{$\begin{array}{l}\text { La psicoterapia genera } \\
\text { crecimiento personal } \\
\text { en el terapeuta. }\end{array}$} & \multirow{5}{*}{$\begin{array}{l}\text { El ejercicio de la psicoterapia } \\
\text { genera un crecimiento } \\
\text { personal y profesional en el } \\
\text { terapeuta, ya que a partir de } \\
\text { la experiencia de ser } \\
\text { paciente, ser supervisado por } \\
\text { psicoterapeutas expertos y } \\
\text { aprender de los pacientes, } \\
\text { implica el desarrollo de un } \\
\text { cuerpo de valores humanos, } \\
\text { orientados a la ayuda del otrc } \\
\text { y a la transformación } \\
\text { personal positiva. }\end{array}$} & A través de la psicoterapia personal. \\
\hline & & & $\begin{array}{l}\text { Mediante el proceso de supervisión } \\
\text { del terapeuta. }\end{array}$ \\
\hline & & & $\begin{array}{l}\text { Años de servicio causa un efecto } \\
\text { positivo en el terapeuta. }\end{array}$ \\
\hline & & & Desarrollo de valores humanos. \\
\hline & & & Búsqueda de transformación. \\
\hline
\end{tabular}

En las Tablas 4, 5, y 6, se presentan fragmentos de texto que dan soporte a las propiedades descubiertas en cada una de las categorías subsidiaras 3 .

Tabla 4 - Sub-categoría "La psicoterapia depende del establecimiento de una buena alianza terapéutica"

\begin{tabular}{|l|l|}
\hline Propiedad & Texto de referencia \\
\hline $\begin{array}{l}\text { Genera una } \\
\text { conexión } \\
\text { profunda. }\end{array}$ & $\begin{array}{l}\text { E7:"hay un encuentro humano y que es necesario que se establezca una alianza real de trabajo, de } \\
\text { cooperación entre el terapeuta y el paciente...sin duda la relación terapéutica es un elemento esencial } \\
\text { porque si no se establece una relación terapéutica, un vínculo, una alianza de trabajo adecuado etc.... } \\
\text { la cosa no va para ningún lado..." }\end{array}$ \\
\hline $\begin{array}{l}\text { Permite que el } \\
\text { paciente se } \\
\text { muestre a } \\
\text { profundidad. }\end{array}$ & $\begin{array}{l}\text { E7: "como el hecho del que paciente comienza a actuar allí su patrón de interacción, como lo quieras } \\
\text { llamar, sus esquemas interpersonales o como usted quiera llamarlo...también comienza un poco en ese } \\
\text { escenario a mostrarse aspecto del funcionamiento del paciente fuera del consultorio" }\end{array}$ \\
\hline $\begin{array}{l}\text { Está basada en la } \\
\text { empatía. }\end{array}$ & E7"para mi tiene que estar basada en la empatía, humildad, respeto, compromiso, responsabilidad" \\
\hline
\end{tabular}

Tabla 5 - Sub-categoría "La psicoterapia favorece el surgimiento de aspectos intimos y subjetivos del paciente"

\begin{tabular}{|c|c|}
\hline Propiedades & Texto de referencia \\
\hline $\begin{array}{l}\text { Siente la } \\
\text { necesidad de } \\
\text { buscar ayuda. }\end{array}$ & $\begin{array}{l}\text { E7: "la psicoterapia es un proceso donde la persona siente como la necesidad de buscar ayuda... en la } \\
\text { mayoría de los casos tienen que ver con que la persona experimenta algún tipo de malestar algo que } \\
\text { considera como un problema en su vida y que es inexplicable para ella y no lo puede entender bien o que } \\
\text { su explicaciones le son insuficientes para poder lograr sentirse bien, es decir, creo que lo que motiva a } \\
\text { la mayoría de los pacientes como la necesidad de lograr algún tipo de cambio en su manera de pensar, } \\
\text { de sentirse, de actuar en fin..." }\end{array}$ \\
\hline $\begin{array}{l}\text { Permite al } \\
\text { Terapeuta conocer } \\
\text { las } \\
\text { vulnerabilidades } \\
\text { del paciente. }\end{array}$ & $\begin{array}{l}\text { E2:"la otra persona que está allí desde la humildad inclusive desde la vulnerabilidad porque te está } \\
\text { mostrando su ser en el momento más compasivo de todos, porque te está mostrando ese ser herido que } \\
\text { no sabe cómo solucionar una situación" } \\
\text { "ese proceso de auto-reconocimiento o de incluso muchas veces de rescate porque a veces la persona } \\
\text { llega totalmente perdida de sí... perdida de su ser, y eso es lo que nosotros tenemos que acompañar a } \\
\text { que ella o que el vuelva a encontrar o retomar, para que entonces a partir de allí ver con que cuentas } \\
\text { para poder solventar esta situación..." }\end{array}$ \\
\hline $\begin{array}{l}\text { El paciente hace } \\
\text { introspección. }\end{array}$ & $\begin{array}{l}\text { E7:"el terapeuta tiene también el deseo de ayudar a la persona a no solamente a lograr este alivio más } \\
\text { extremo inmediato que es lo que la personas buscan, sino también a conocerse y a descubrir que detrás } \\
\text { de ese síntoma, detrás de esa sintomatología que lo afecta poder descubrir cosas esenciales de sí mismo } \\
\text { que lo ayuden, no solamente a sentirse mejor... porque ciertamente en parte creo que la responsabilidad } \\
\text { tiene que ver con ayudar a la persona a lograr algunos cambios pero principalmente como a descubrirse, } \\
\text { aceptarse en un muchos aspectos y a tener una condición que le permita vivir lo mejor posible, lo más }\end{array}$ \\
\hline
\end{tabular}

\section{CeIR Vol. 13 (2) - Octubre 2019 ISSN 1988-2939 - www.ceir.info}

(c) Derechos reservados/Copyright de Clínica e investigación Relacional y los autores. Prohibida la reproducción total o parcial sin autorización expresa. Este material es para uso científico y profesional exclusivamente y puede contener información clínica sensible. Los editores no se responsabilizan de los contenidos de los autores. Dirigir las consultas sobre derechos y autorizaciones a ceir@psicoterapiarelacional.es 


\begin{tabular}{|l|l|}
\hline & $\begin{array}{l}\text { funcional posible porque creo que no está dirigidos solamente al alivio de ese malestar inmediato que } \\
\text { suele traer a las personas a consulta donde algunas personas pueden tener también deseos de } \\
\text { conocerse más" }\end{array}$ \\
\hline $\begin{array}{l}\text { Es un proceso que } \\
\text { genera } \\
\text { transformaciones } \\
\text { o cambios } \\
\text { positivos }\end{array}$ & $\begin{array}{l}\text { E2 porque una persona puede transformar su vida sin cambiarla ok, o yo puedo ser una mejor versión } \\
\text { de mín }\end{array}$ \\
\hline
\end{tabular}

Tabla 6 - Sub-categoría "La psicoterapia genera crecimiento personal en el terapeuta"

\begin{tabular}{|l|l|}
\hline Propiedades & Texto de Referencia \\
\hline $\begin{array}{l}\text { A través de la } \\
\text { psicoterapia } \\
\text { personal. }\end{array}$ & $\begin{array}{l}\text { E4:"...este, a nivel personal ver tantas cosas en mi como analizante, a nivel personal... a nivel } \\
\text { profesional imagínate... es que cuando trabajas a nivel personal, te ayuda a nivel profesional, ¿me } \\
\text { explico?... porque bueno la neurosis no te deja saber, no te deja desenvolverte, o sea que son tantas } \\
\text { cosas, que dándote cuenta con la terapia, es que lo puedes trabajar..." }\end{array}$ \\
\hline $\begin{array}{l}\text { Mediante el } \\
\text { proceso de } \\
\text { supervisión del } \\
\text { terapeuta. }\end{array}$ & $\begin{array}{l}\text { E6:" el terapeuta tiene que estar trabajando constantemente bajo supervisión porque en proceso } \\
\text { terapéutico tiene que haber los 3 ojos, 1 hacia el paciente, 1 hacia el proceso, 1 hacia sí mismo". }\end{array}$ \\
\hline $\begin{array}{l}\text { Años de servicio } \\
\text { causa un efecto } \\
\text { positivo en el } \\
\text { terapeuta. }\end{array}$ & $\begin{array}{l}\text { E7:":"bueno yo creo que he aprendido en particular a ser más flexible creo, he creo que la psicoterapia } \\
\text { tiene que ver con relacionarse con distintos tipos de personas, tiene que ver con adaptarse a diferentes } \\
\text { tipos de personas y eso ínsita a uno a la flexibilidad entender que no" }\end{array}$ \\
\hline $\begin{array}{l}\text { Desarrollo de } \\
\text { valores humanos. }\end{array}$ & $\begin{array}{l}\text { E6: "el respeto y la dignidad eso para mí es fundamental, por eso tengo que ser así no puedo ser de } \\
\text { otra manera, no puedo trabajar desde otro enfoque" } \\
\text { "para mi tiene que estar basada en humildad, respeto, compromiso, responsabilidad..." }\end{array}$ \\
\hline $\begin{array}{l}\text { Búsqueda de } \\
\text { transformación. }\end{array}$ & $\begin{array}{l}\text { E7: "el trabajo en psicoterapia me ayudado a no solamente en... en realizar, en mirarme, sino que en } \\
\text { para mí ha sido un importante recurso también para protegerme, pero protegerme no sé si es la } \\
\text { palabra, pero para he muchas veces sanamente desconectarme de la realidad" }\end{array}$ \\
\hline
\end{tabular}

\section{Descripción del proceso de representación gráfica}

Una vez definida y sometida a discusión, las categorías entrelazadas pasaron a ser graficadas (Figura 1) y su lectura produjo un relato o construcción teórica, llamada por Strauss y Corbin (2002), matriz condicional o consecuencial. A partir de este paso final se pudo formular la concepción de la psicoterapia en el psicólogo clínico venezolano. Las relaciones, las categorías y sus propiedades se describen en lo sucesivo con el fin de reducir la teoría implícita emergente en cada fragmento y luego integrarla en el análisis concluyente. En la Figura 1, las relaciones directas se simbolizan con flechas gruesas y las indirectas con flechas punteadas. 
Figura 1 - Relaciones entre las categorías y sus propiedades

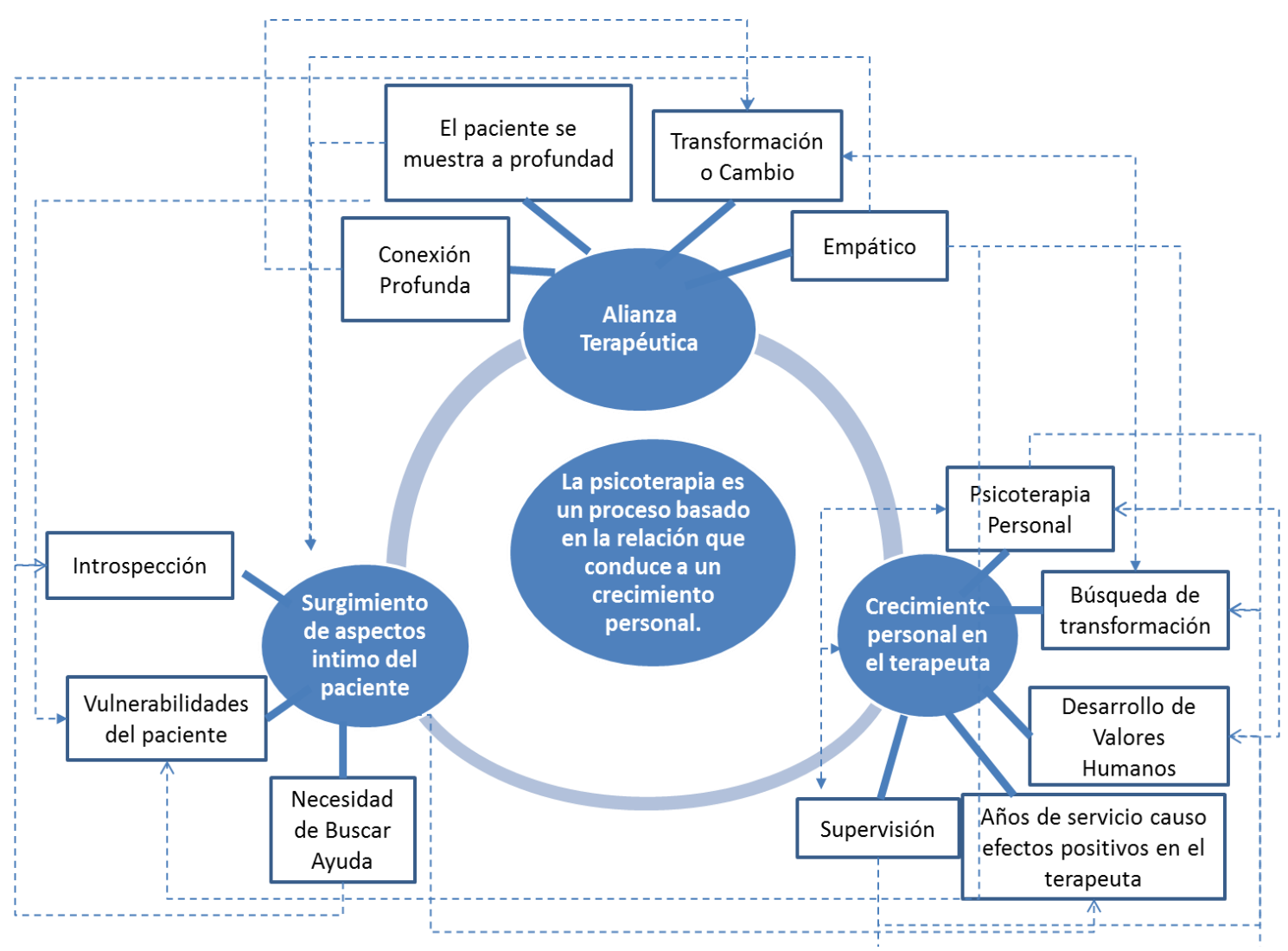

\section{ANÁLISIS DE LOS RESULTADOS}

A continuación, se presenta un análisis exhaustivo de cada categoría, se desglosa su significado y se ejemplifica con verbalizaciones textuales aportadas por los informantes en las entrevistas 4 .

Sub-categoría: La psicoterapia depende del establecimiento de una buena alianza terapéutica

La psicoterapia es un procedimiento que está basado en el establecimiento de una relación terapéutica, que depende de una alianza y un vínculo, que genera una conexión profunda entre el terapeuta y paciente, que va a permitir el proceso de acompañamiento.

En psicoterapia, para que la alianza terapéutica pueda ser sólida, tiene que estar conformada por una serie de características como la empatía, respeto, autenticidad, lealtad, responsabilidad, confianza, que en su gran mayoría engloba el marco ético del terapeuta. 
Y es en el vínculo donde el paciente se muestra, es decir, deja ver al terapeuta su self y eso va a permitir que el mismo comience a generar transformaciones o cambios, que determinaran en buena parte su manera de relacionarse con las personas de manera diferente, más adaptativo y saludable.

Sub-categoría: La psicoterapia favorece el surgimiento de aspectos íntimos y subjetivos del paciente

El paciente siente la necesidad de buscar ayuda, debido a que tiene un problema, síntoma o un padecimiento que le causa un malestar psicológico; esta razón motiva al paciente asistir a consulta para encontrar un alivio a su sufrimiento, pero posteriormente le va a permitir hacer una introspección en su vida y extrapolar lo aprendido en el proceso psicoterapéutico a otras áreas y contextos.

En este contexto es importante acotar que dentro del proceso psicoterapéutico van a surgir aspectos íntimos y también subjetivos de cómo esta persona ha construido su self, que le permitirán al terapeuta ver las vulnerabilidades del paciente.

\section{Sub-categoría: La psicoterapia genera crecimiento personal en el terapeuta}

En el psicólogo clínico, la psicoterapia conlleva a sentir a que su crecimiento personal y profesional va a estar constituido por su psicoterapia personal:

E6: "...y que pasa que muchísimos de nosotros a veces de manera irresponsable, hasta nos jactamos de que nunca fuimos a terapia... yo llevo 33 años y son los mejores reales invertidos, no sé si tendré salud mental pero lo gozo una bola, jajaja, cuando voy a terapia, y siento que es importante entender que lo neurótica que estoy, o sea eso es inevitable"

Se considera que parte importante del crecimiento profesional del terapeuta es el proceso de supervisión, porque permite ver la posición ante el paciente y a su vez ayudar al mismo a trabajar esa posición a través de la psicoterapia personal, es decir, que estos dos procedimientos son esenciales en la formación del psicoterapeuta.

La psicoterapia obliga al psicólogo clínico al desarrollo de un cuerpo de valores humanos, que facilitaran el proceso de conexión, y también a no juzgar al paciente:

E4:"...La atribución tiene mucho que ver, 1er con el buen trabajo y la evaluación terapéutica, el compromiso, el manejo de concepto de la dignidad, responsabilidad y que no es una relación posicional, y que el otro no te pertenece... es la vida del otro, es la autonomía del otro... y es fomentar esa autonomía, no la dependencia..."

Se considera que los años dedicados al ejercicio de la psicoterapia tienen un efecto positivo en la vida del psicólogo clínico, ya que trae consigo el crecimiento en muchos aspectos de su vida: 
E7: "el trabajo en psicoterapia me ayudado a no solamente en... en realizar, en mirarme, sino que en para mí ha sido un importante recurso también para protegerme, pero protegerme no sé si es la palabra, pero para he muchas veces sanamente desconectarme de la realidad".

Finalmente, la psicoterapia genera en el psicólogo clínico una búsqueda a la transformación, desde un encuentro con el otro, desde su propia vida íntima, terapia personal y desde la formación, permitiendo un cambio significativo:

E5:"...y con los años se aprende el gozo que termina cuando tu logras controlar el sufrimiento, logras hacerlo parte de ti y de tu protector..."

Es importante resaltar que en el proceso de recolección de datos y en el análisis de las entrevistas, surgieron ideas que, aunque no se categorizaron, resultan importantes en el análisis, particularmente aquellas relacionadas con el hecho de que la elección de esta profesión tiene que ver con características personales, con la historia de vida, con la dinámica de familia y el deseo inconsciente de saber qué sucede en la familia y a su vez resolverlo.

Tenemos entonces algunas verbalizaciones de las razones que aducen los psicólogos clínicos que influyeron al haber escogido esta profesión.

El querer ayudar a los demás:

E5: "El amor por la gente... yo siempre he sido muy compasiva... o sea me gusta la gente...."

Desde la historia de vida:

E7: :"... yo creo que tiene que ver con la historia de uno... mi caso particular es que he bueno hay, mi papa murió muy joven yo siempre fui como el apoyo de mi mama, soy el hermano mayor, el que escuchaba siempre los cuentos de mi mama, mi posición en la vida siempre ha sido como desde muy temprano como de ser el que escucha y apoya... y yo creo que era razonable que escogiera algo que tiene que ver con escuchar y apoyar, eso por un lado..."

Por la dinámica de familia:

E4:"con la historia familiar, bueno porque yo pienso que siempre hay algo como que nos mueve, que no anda muy bien... que uno quiere, ver que pasa allí... yo como que muchos desencuentros con mi mama, entonces eso... era como que me daba mucho malestar, mucho malestar... muchos desencuentros, muchos desencuentros... muy apegada a mi papa, yentonces yo pienso que eso como que también aja... hace preguntas de alguna manera... y uno dice voy ayudar al otro... pero después que uno empieza a estudiar la carrera, es como que: jaaaah!, esto me pasa, esto lo tengo yo, esto tal... yo pienso que también por ahí también va..."

Dentro del análisis de los datos de esta investigación, se encontraron incidencias considerables que resultaron negativas en cuanto al proceso de la psicoterapia, específicamente las tienen que ver con la iatrogenia: 
E6: "para mi es el vínculo, yo siempre digo que un terapeuta... puede ser el peor aplicando técnicas pero que si tiene un buen vinculo establecido con el paciente, o sea si hay una buena sintonía, si hay un buen trabajo, el respeto, sino se violenta, sino se actúa el clisé"

El psicólogo clínico puede poner en riesgo el proceso de psicoterapia si no existe la capacidad de introspección, que conlleva a trabajar en su propio proceso personal, y a su vez, en buena parte el trabajo supervisado, para identificar desde dónde ve el terapeuta al paciente. De acuerdo a lo planteado por los participantes, la psicoterapia tiende a crear encuentros de crecimiento dirigidos al paciente, para favorecer su autonomía empoderarle en el contexto relacional que lo rodea:

E5: "entonces esto igual que en la música tiene que haber un profundo respeto por el tiempo musical, también tiene que haber un profundo respeto por el tiempo de las personas, ¿entiendes?... es decir, dejarlo ser, digamos... vivir pero no dejar morir, jajaja... entonces déjalo que vaya viviendo pero no lo dejes morir tampoco, no... entonces esta vivirá, de lidear con el tiempo del paciente, y no perderle el tiempo al paciente, es decir, no es que lo dejas muchísimo tiempo ahí, muriéndose sino que de alguna manera tenga presente que hay que hacer algunas intervenciones que se hagan que avance la persona, pero siempre respetando profundamente su ritmo, no... entonces esta es otra cosa que en general los terapeutas Venezolanos,... bueno psicoterapeutas Venezolanos hacen. Las 3 cosas más importante que en mi opinión un terapeuta venezolano depende... 1ero dar permiso de dejar crecer al paciente, 2 do que ese permiso sea con potencia, o sea que lo empodere y zero que le proteja, que lo proteja de cualquier cosa..."

La psicoterapia es un procedimiento que potencia la salud mental, ya que se produce un vínculo entre terapeuta y paciente, y se espera que ese vínculo sea lo más útil, realista, y cercano al mundo exterior que vive el paciente en constante contacto:

E7: "sin duda la relación terapéutica es un elemento esencial porque si no se establece una relación terapéutica, un vínculo, una alianza de trabajo adecuado etc.... la cosa no va para ningún lado... que, además la relación terapéutica permite que el paciente muestre la relación, su funcionamiento y aspectos de un elemento de afuera..."

\section{Análisis de las coordenadas más importantes}

Fundamentada en los datos, pasamos a describir las coordenadas teóricas más importantes que se derivan de las verbalizaciones de los informantes.

\section{Definición de la psicoterapia en el psicólogo clínico venezolano}

Es un proceso que se logra con del establecimiento de una relación, a través de una buena alianza terapéutica, que va a permitir el surgimiento de aspectos íntimos del paciente, ya que permite ver la subjetividad del paciente y a su vez genera un crecimiento personal en el terapeuta.

Esta alianza terapéutica está basada en el proceso de acompañamiento, que va a desarrollar un vínculo profundo y que permitirá el crecimiento personal del paciente. La formación de 
esta alianza y el vínculo dependerá de una empatía sincera por parte del terapeuta, de allí surge la conexión profunda entre el paciente y terapeuta.

\section{La psicoterapia muestra el ser del paciente}

La psicoterapia es un espacio donde llega el paciente a buscar ayuda por un sentimiento de malestar subjetivo; partiendo desde ese punto, permitirá mostrar aspectos íntimos y subjetivos, que ocasionan introspección y el cambio o transformación de sí mismo. La psicoterapia permite conocer al terapeuta cuales son las vulnerabilidades del paciente y existe una gran probabilidad que los conflictos internos del paciente se manifiesten en la relación con el terapeuta.

\section{La psicoterapia genera crecimiento personal en el terapeuta}

El ejercicio de la psicoterapia ocasiona un crecimiento personal y profesional, ya que el terapeuta a partir de la experiencia de ser paciente, de ser supervisado y en el hecho de aprender de los pacientes desde ese encuentro entre dos, permite desarrollar los valores humanos que están orientados hacia la ayuda del otro y hacia la transformación o cambio personal de manera positiva.

\section{La psicoterapia personal produce cambios}

La psicoterapia personal del terapeuta produce contacto consigo mismo, y esto le permitirá conocer sus vulnerabilidades, para no solamente crecer desde esa posición, sino también la de ser supervisado, para realizar una autoevaluación de su posición ante cada paciente y de la forma de relacionarse, obteniendo la capacidad de transformar su vida.

\section{Psicoterapia personal y supervisión}

Freud (1912) en Consejos al médico en el tratamiento psicoanalítico, menciona que el análisis personal es una exigencia para los analistas que desean tratar pacientes en psicoterapia y donde es posible revisar sus puntos ciegos. Ante este texto de referencia, tiene en concordancia con una de las ideas obtenidas en esta investigación: que la psicoterapia personal es necesaria para la formación como terapeuta y también para que se dé el autoconocimiento de si y de sus vulnerabilidades por parte del terapeuta.

\section{Características culturales que favorecen la Psicoterapia}

Alejandro Moreno (1999) refiere que la antropología del venezolano popular, a partir de la estructura matricentrada de la familia típica predominante en Venezuela, se sostiene sobre la constitución relacional. Explica que el venezolano popular no puede ser pensado ni concebido como individuo, sino como "relación estructural". La "relación convivencial" que lo hace "ser en relación", condiciona en profundidad toda su postura. Al respecto, ha 
señalado este autor lo que parece debe ser, a partir-del-mundo popular, la forma de ejercer cualquier tipo de orientación y psicología clínica en Venezuela, debe ser esencialmente relacional, esto es, centrado en la relación personal en la que vive el venezolano. El psicólogo y orientador en el ejercicio de la orientación tiene que partir ya que la relación está presente, aunque se encuentre retraída. Se trata de manejarla y facilitar su dimensión amorosa, no de producirla.

Las coincidencias de Alejandro Moreno con los resultados de este estudio son significativas, debido a que demuestra que el venezolano tiene la capacidad de acceder al proceso de psicoterapia basada en la relación, ya que tiene como huella cultural la capacidad (y necesidad) de vinculación, constituyéndose un "self relacional" consustancial con el modo de ser venezolano.

\section{Coordenada central: la persona del terapeuta define la concepción de la psicoterapia}

La psicoterapia es un proceso basado en un tipo especial de relación que conduce a un crecimiento personal. Es un proceso que se logra a través del establecimiento de una especial relación, que permite acceder a la subjetividad del paciente y que genera un crecimiento personal del propio terapeuta. La psicoterapia depende del establecimiento de una buena alianza terapéutica.

La relación terapéutica elación que está basada en el establecimiento de un vínculo y una alianza que permite conocer al paciente; esta relación favorece el surgimiento de aspectos íntimos y subjetivos del paciente. Es un espacio donde llega la persona a consultar o buscar ayuda a causa de problemas o padecimientos que lo lleva al sufrimiento, y es en ese proceso donde este paciente muestra su ser y a su vez permite conocerse a sí mismo y así poder construir un cambio o transformación en su vida.

El ejercicio de la psicoterapia genera un crecimiento personal y profesional en el terapeuta, ya que, a partir de la experiencia de ser paciente (es decir, su propio proceso personal de psicoterapia), ser supervisado por psicoterapeutas expertos y aprender de los pacientes, implica el desarrollo de un cuerpo de valores humanos, orientados a la ayuda del otro y a la transformación personal positiva

Por otro lado, el terapeuta tiene unas características únicas dentro de todos los profesionales, de acuerdo a lo que se encontró en esta investigación, su self es un self que está centrado en ayudar y acompañar al otro, y esta persona ha desarrollado estas características y por ende eso tiene que ver con su historia personal; de manera que el sentirse útil, ayudar a otro, y eso desarrolla un self de ayudar; y esta persona cuando despliega ese self se siente bien; generando pasión motivacional en el terapeuta de explorar, 
siente la curiosidad por el saber, el enigma por la vida o por el mundo del otro, y desde allí le da respuestas, en soluciones e integra las experiencia del paciente.

En esta investigación se ha evidenciado que no solamente el enfoque es lo que define el éxito o la efectividad de la psicoterapia, también la personalidad del psicoterapeuta, el "cómo" se apropia de los conocimientos y la formación y de la significación que tiene el proceso terapéutico personal.

El psicoterapeuta es la terapia, ya que él conoce, maneja y comprende su importancia, evidenciándose la necesidad de la supervisión, porque puede obtener como consecuencia negativa, justificar su vida o su self, independientemente del nivel de ganancia económica que tenga; además, los informantes dejaron en claridad la importancia que tiene la supervisión, es decir, que tienen conciencia del daño potencial que pueden causar al paciente si no supervisan sus propias reacciones subjetivas.

El poder puede ser una motivación en el terapeuta, para estar plenamente en ese proceso terapéutico, desplegar todos sus recursos personales y teóricos, ya que existe una conciencia de la importancia que tiene en el proceso del otro; además genera un nivel de entrega tan profundo, que cuando no existen cambios, o el proceso terapéutico atraviesa por una etapa de obstaculización o estancamiento, desciende ese poder, ocasionando una perdida narcisista y allí puede generar una contratransferencia negativa o no útil. De esta manera, se reafirma la importancia de la supervisión y de la psicoterapia personal, para que haya un manejo de poder positivo y ético.

Otro aspecto importante, es que el ejercicio de la psicoterapia puede implicar un medio de escape de la vida personal del terapeuta; el tiempo en el que el terapeuta está atendiendo a un paciente, deja de verse a sí mismo, y de alguna manera u otra, le ofrece un descanso a toda la problemática personal o del entorno en general que pueda tener el terapeuta; también puede generar una compensación de afectos que faltan en la vida personal del terapeuta, y eso puede convertirse en algo iatrogénico para el paciente y también para el terapeuta. Sin embargo, se evidencia que es un escape fallido en el proceso psicoterapéutico, se toca el self y la vida del terapeuta tangencialmente, y esto puede ser un decrecimiento, y debe trabajarse en la psicoterapia personal del terapeuta.

Guy (1987), refiere las motivaciones disfuncionales con respecto a la elección de ser psicoterapeuta, corresponde a motivaciones que si bien podrían ser funcionales al ser llevadas al extremo entorpecen tanto la vida personal como profesional de los terapeutas, el autor reconoce las siguientes: aflicción emocional (necesidad de autocomprensión, asociado a la necesidad de resolver problemas personales o traumas emocionales), enfrentamiento indirecto (la psicoterapia como medio para afrontar indirectamente las contingencias de la 
vida a través de la experiencia del paciente), soledad y aislamiento (ser terapeutas como un intento de superar ese sentimiento), deseo de poder (tendencia general hacia la agresividad, dominio o siente necesidad de influir, controlar y puede ocasionar dificultad al momento de respetar el derecho y la responsabilidad del paciente en si propia opinión y autonomía), necesidad de amor (poder expresar amor y ternura en un espacio seguro) y como una forma de rebelión indirecta. Estas dimensiones se evidencian de modo significativo en una de las verbalizaciones de los informantes:

E1: "yo creo que lo más iatrogénico en un proceso de psicoterapia es que el terapeuta ponga su narcisismo.... Es decir, que tu narcisismo se satisfaga... o sea una cosa es que tu disfrutes tu trabajo, que te admire que el paciente cambie y que tu cambies, producto del paciente... digamos no... pero otra cosa muy distinta es que tu pongas tus necesidades narcisistas... la vulnerabilidades siempre van a estar alli... pero que tu digamos... sin darte cuenta o intencionalmente, pongas tu narcisismo... Pongas tus necesidades de ser querido, de ser afectado, de ser valorado en la relación terapéutica... eso yo creo que es muy iatrogénico..."

Por otra parte, el efecto de los años de experiencia, aumenta su respeto y admiración, por el paciente, respetando su ritmo y su historia; aumenta el respeto por sí mismo y su autocuidado; despliega sus normas personales, también su estilo propio, de acuerdo a su experiencia, aumentando la flexibilidad en cuanto al encuadre de la relación terapéutica y apertura hacia la posición del terapeuta, que pueda sentirse cómodo y seguro de sí mismo, esto significa que aumenta su capacidad de entrega, y a su vez también se hacen más humildes, auténticos, se muestran más, usándose a sí mismos como parte del proceso; se aprende a no ser arrogante, conllevando a ser tiernos y más iguales al paciente, a pesar de que es una relación asimétrica. También delegan más el poder al paciente, es decir, en el proceso de la psicoterapia, le modela al paciente a empoderarse de su propia vida.

E5: "las 3 cosas más importante que en mi opinión un terapeuta venezolano depende... 1ero dar permiso de dejar crecer al paciente, 2 do que ese permiso sea con potencia, o sea que lo empodere $y$ zero que le proteja, que lo proteja de cualquier cosa..."

Otro aspecto importante, es que disminuye la rivalidad entre terapeutas, al descubrir que cada quien tiene su espacio, y que hay diferentes estilos de terapeutas, para una población especifica de paciente, según la comodidad del mismo, y que no significa precisamente una perdida narcisista, sino que permite darse cuenta, que un terapeuta no funciona para todo, y eso implica el respeto de sí mismo, y hacia al paciente:

E7: "... por lo menos desde el punto de vista de la alianza se establezca a mi juicio depende de tres cosas: la personalidad del paciente y hay va incluido su expectativa, etc... su forma de ser... la personalidad del terapeuta y el encaje que hagan los dos, quiere decir... al final no es que la personalidad del terapeuta va a determinar el éxito de la relación, por ejemplo, sino que esa personalidad influirá o no dependiendo también de la personalidad del paciente, es decir, hay 
terapeuta para determinado tipos de paciente o hay pacientes para determinado tipos de terapeuta... o sea, el tema es el encaje..."

Finalmente, los años de experiencia implican grandes cambios en el psicoterapeuta, debido a que aumenta el amor y respeto por el ejercicio de la profesión y la gratitud por ejercerla. También crece la entrega, la responsabilidad por las vidas que toca y se desarrolla la capacidad de reconocimiento y amor hacia el paciente.

\section{A modo de cierre}

En esta investigación, se preguntó cómo concibe el psicólogo clínico venezolano a la psicoterapia. Para responder a esta pregunta, se propuso indagar, desde las propias vivencias fenomenológicas de los mismos psicólogos, cómo entienden ellos este procedimiento. Utilizando el enfoque cualitativo (entrevistas) y desde la Teoría Fundamentada, se trató de dar cuenta de esta pregunta. Se propuso un esbozo de teoría, que, si bien es exhaustivo y profundo, necesita de mayor representatividad para que sus resultados puedan generalizarse a toda la población de psicólogos en Venezuela. La concepción de la psicoterapia como un vínculo profundo único, especial y sobre todo humano con el paciente, fue una de las respuestas trasversales en todos los sujetos entrevistados. Pareciera que este descubrimiento da cuenta de investigaciones llevadas a cabo desde un paradigma antropológico, realizado por otros autores (e.g. Moreno. 1999), sobre el modo de vida relacional, en el cual se encuentra inmerso el venezolano. Quizás la combinación de métodos cuantitativos con los cualitativos descritos en esta investigación, amplien la respuesta a la pregunta inicial.

\section{REFERENCIAS}

Amaro F., Guasch L.L., y Sarlé M. (2007). "Psicoterapia individual intensiva psicodinámica: La relación terapéutica y las técnicas de interpretación". Universidad Autónoma de Barcelona. Colegio Oficial de Psicólogos. Recuperado de http://www.paidopsiquiatria.cat/files/modulo9.Psicoterapia_individual intensiva_psicodinamica la relacio o.pdf. 24 de marzo de 2019

Asociación Venezolana de Psicoterapia (AVEPSI). Recuperado de: http://avepsi.org.ve/site/la asociacion/historia-de-avepsil. 13 de abril de 2019

Freud S. (1912). Consejos al médico sobre el tratamiento psicoanalítico. En Obras completas. Buenos Aires, Argentina, Amorrortu Editores. ISBN 978-950-518-575-7.

Gómez, M. (2010). Concepto de psicoterapia en psicología clínica. Revista de Psicología Universidad de Antioquia, 2(2), 19-32. Recuperado de http://pepsic.bvsalud.org/scielo.php?script= sci_arttext\&pid=S214548922010000200003\&lng=pt\&tlng=es 14 de febrero de 2019.

Guy, J. (1995). La vida personal del psicoterapeuta: el impacto de la práctica clínica en las emociones y vivencias del terapeuta. Barcelona, España: Paidós 
Hernandez, R. Fernandez C., y Baptista L. (2006). Metodología de la Investigación. McGraw-Hill. México (D.F.), México: Cuarta Edición

Lilliengren, P. y Werbart, A. (2010). A therapists' view of therapeutic action in psychoanalytic psychotherapy with young adults. Psychotherapy Theory, Research, Practice, Training Vol. 47,4, 570-585

López L. (2010). La otra cara de la terapia: la vida personal del Psicoterapeuta. Revista Electrónica de Psicología Social «Poiésis» No 19. ISSN 1692-0945. Recuperado de http://www.funlam.edu.co/poiesis 13 de diciembre de 2018

Moreno, Alejandro (1999). La familia en el ejercicio de la orientación y el asesoramiento. Heterotopía 11

Rodríguez P. R. C. y Sánchez, L. M. (1996). La psicología en Venezuela. Papeles del Psicólogo. Vol. (66)

Strasbourg Declaration on Psychotherapy of 1990. Recuperado de: https://www.europsyche.org/contents/13247/strasbourg-declaration-on-psychotherapy-of-19go. 15 de enero de 2019

\section{Anexo 1 - Preguntas significativas y más repetidas durante las entrevistas}

1. ¿Qué es para usted la psicoterapia? Hablando de eso, entonces para usted ¿qué sería un proceso terapéutico?

2. ¿Cuál es su formación específica en psicoterapia?

3. ¿Qué cree usted que hace efectiva a la psicoterapia?

4. Veo entonces que usted le da mucha importancia a la relación, al vínculo terapéutico que se pueda establecer entre el terapeuta y paciente. ¿Cómo podría elaborar esto?

5. Háblame un poco de esa relación terapéutica y la importancia que usted le otorga al ejercicio de la psicoterapia. Desde su punto de vista, ¿cómo es la relación terapéutica?

6. Háblame un poco, de sus años de experiencia y cómo usted ha ido concibiendo el concepto de psicoterapia. ¿Qué le ha enseñado a usted los años de experiencia en psicoterapia?

7. Háblame de su Self, es decir, ¿usted ha visto cambios en usted mismo al hacer psicoterapia?

8. Y usted, desde su historia personal, ¿por qué decide escoger esta profesión como psicoterapeuta?

9. Para usted, ¿qué tan importante es la personalidad del terapeuta?

10. Para usted, ¿qué puede ser iatrogénico para la terapia?

11. Cómo interpreta usted los casos, ¿dependiendo de la persona y la realidad del paciente?

12. Para usted, es importante la formación, la supervisión tanto del trabajo como terapeuta y la supervisión individual. ¿Podría elaborar esto un poco más?

13. Y desde su historia de vida, ¿por qué escogió estudiar esta profesión? 
Original recibido con fecha: 31/3/2019 Revisado: 30/09/2019 Aceptado: 30/09/2019

NOTAS:

${ }^{1}$ Aunque se deje en claro que los psicólogos clínicos no son los únicos profesionales que llevan a cabo labores psicoterapéuticas en Venezuela, si se destaca que éstos son quienes generalmente la emplean como medio de tratamiento de las dificultades y/o trastornos relacionados con la conducta.

${ }^{2}$ La Declaración de Estrasburgo, realizada en Estrasburgo (Francia) el 21 de octubre de 199o, establece que la psicoterapia es una disciplina científica independiente, y su ejercicio equivale a una profesión libre y autónoma; la formación del psicoterapeuta se debe realizar a un nivel altamente calificado y científico, en el cual se garantizará la diversidad de los métodos psicoterapéuticos de base empírica y científicamente comprobados. Así mismo, se dejó claro en esta declaración que el proceso de entrenamiento abarca la formación teórica, la práctica clínica acompañada de supervisión y la terapia personal, y se realiza por completo en el marco de un mismo método psicoterapéutico, al tiempo que se adquirieren suficientes conocimientos de otros métodos psicoterapéuticos; el acceso a la formación se efectúa a través de diferentes calificaciones preliminares y en particular, a través de las ciencias humanas y sociales (Strasbourg Declaration on Psychotherapy of 1990 https://www.europsyche.org/contents/13247/strasbourg-declaration-onpsychotherapy-of-19g0).

3 Debido a que esta es una investigación pionera en Venezuela, los investigadores acordaron la inclusión de al menos 1 reporte textual coincidentes que dieron origen a la categorización. Los fragmentos se identifican en cursiva, según el número del entrevistado, y se denominan $E_{1}, E_{2}, E_{3}, E_{4}, E_{5}, E 6$, E7, en función de los 7 informantes.

${ }^{4}$ Los fragmentos textuales se identifican en cursiva, según el número del entrevistado, y se denominan $E_{1}$, $E_{2}, E_{3}, E_{4}, E_{5}, E 6, E 7$, en función de los siete informantes 